\title{
Informing the next R\&D generation: The Erice School on Materials for Renewable Energy and Sustainability
}

\author{
www.erice-energy-materials2018.ct.infn.it
}

$\mathrm{T}_{\mathrm{s}}^{\mathrm{h}}, \mathrm{mes}$ he MRS-E-MRS International School on Materials for Renewable Energy and Sustainability, which started in 2010 at the "Ettore Majorana" Foundation and Centre for Scientific Culture (Erice, Italy), has completed yet another successful session.

The School has been held in Erice during even-numbered years since 2015, and is held in the United States during odd-numbered years. Thanks to a Memorandum of Understanding between MRS and E-MRS, at least three more schools will be organized through 2021. With an average number of students of $\sim 70$, a total of about 500 people from more than 30 nations have shared this unique experience to learn about the key roles of energy and materials for a sustainable world. Along with the students, more than 50 lecturers from leading academic and industrial institutions worldwide have taught at these schools, providing a great learning opportunity for the next generation of researchers.

The Erice School this year was a full immersion into materials science and technology applied to the generation, storage, and use of green energy for sustainable development. The school included overviews on global climate, $\mathrm{CO}_{2}$ sequestration, water-energy nexus, nuclear energy, solar thermal and photovoltaic power, as well as photosynthesis and biofuels, fuel cells, wind energy, thermoelectricity, energy storage, the electrical grid, and mobility/transportation from the point of view of energy and sustainability.

Randomized seating increased students' interactions and helped to avoid the formation of small "closed" groups based on native language or scientific background. Another added value was the assignment of questions and simple exercises that were distributed to students a few days prior to the school and discussed at the end of the first day. Two poster sessions, with a total of 52 presentations, provided strong scientific interactions. Finally, as a tradition and grand finale, teams of students, formed again by a random selection of students, were assigned a challenge that needed to be met before the end of the school. This year, the challenge was to build a large building or ship (modeled after existing ones that could be rebuilt from scratch) with zero or negative energy consumption during operation. Teams prepared oral presentations at the conclusion and answered questions and comments from their colleagues and lecturers.

Several public and private institutions within Europe and the United States provided strong financial support. Special acknowledgment goes to the National Renewable Energy Laboratory and the Colorado School of Mines, as well as to the University of Catania and ENEL Green Power, the latter being one of the largest companies in the world for production, distribution, and innovation in the field of energy. More than half the students attended the school thanks to partial or full scholarships.

All of the students and lecturers provided extremely positive feedback, which is the main reason for the continued efforts to keep this school running in the future and finding ways to extend its impact worldwide. To this aim, videos of the lectures have been recorded this year and may be made available on the web shortly.

Antonio Terrasi University of Catania, Italy

Group photo of participants taken at the Erice School in Italy. 